\title{
Electron-Acoustic Phonon Scattering Rates in Spherical Quantum Dots: Contribution of the Macroscopic Deformation Potential
}

\author{
A. M. Alcalde, G. E. Marques, \\ Departamento de Física, UFSCar, Caixa Postal 676, 13565-905 São Carlos SP, Brazil \\ G. Weber, \\ Universidade São Francisco, 13251-900 Itatiba SP, Brazil \\ and T. L. Reinecke \\ Naval Research Laboratory, Washington, D.C. 20375, USA
}

Received on 23 April, 2001

\begin{abstract}
The electron-acoustic-phonon scattering process in spherical II-VI quantum dots is discussed. The quantized acoustic modes are described in terms of the Lamb's classical theory. We considered two mechanism for the interaction between electrons and acoustic modes: microscopic deformation potential, and macroscopic acoustic deformation, also called the ripple mechanism. We also discuss the influence of the glass matrix on the electron-phonon coupling. Our calculations show that the ripple mechanism scattering rates become dominant by more than an order of magnitude, for small dot radius. In general, the total scattering rate depends on the acoustical properties of the glass matrix.
\end{abstract}

The electron-phonon interaction controls a wide range of phenomena such as the cooling of optically excited carriers on the picosecond time scale $[1,2]$. In a quantum dot system, the role of the electron-phonon interaction on a carrier relaxation process has been discussed in terms of possible slowing of relaxation rates due to the effects of confinement, in particular, on the phonon system. The physical mechanisms underlying carrier relaxation are yet not fully understood. In particular there is currently some debate related to the phonon bottleneck effect, especially regarding its apparent absence in small II-VI quantum dots. Therefore, it is necessary that alternative carrier relaxation paths should be discussed systematically. In this work, we calculate the electron-acoustic-phonon scattering rates in $\mathrm{CdS}_{1-x} \mathrm{Se}_{x}$ spherical quantum dots embedded in a glass matrix. The acoustic modes are described in terms of the Lamb's classical theory of the oscillations of a continuous elastic sphere $[3,4,5]$. This model is valid if the wavelengths of the acoustic phonons are sufficiently larger than the lattice constants of the semiconductor. For the Poisson constants considered in this work, this condition is reasonably valid. We have considered two mechanisms for the electron-phonon interaction: The microscopic deformation potential (MDP) and the ripple mechanism (RM) [6, 7], which results from the time dependent modulations of the interface. The effects of the coupling of the phonons of a quantum dot with the surrounding matrix are also discussed here.

In semiconductor nanocrystals, the acoustical modes become discrete due to the size quantization. Their properties can be described in terms of Lamb's classical theory of the oscillations of a continuous elastic sphere. If the nanocrystal shape is supposed to be spherical, Lamb theory predicts the existence of two elastic oscillations leading to torsional and compressional modes. The purely compressional mode has special interest since it provides the dominant contribution to the acoustic phonon deformation potential. We restrict our attention to the Raman active acoustical modes. Duval et al. [4] have determined that only modes with $l=0,2$, can contribute to the Raman scattering of the light in first order. The lowest order spheroidal mode, $l=m=0$, is often called the breathing mode. This mode provides the dominant contribution in the scattering process. The breathing mode displacement inside the dot is purely longitudinal and the dot size dependence of their frequencies $\omega_{k}$ can be calculated by considering the influence of the surface on the phonon displacements. This influence is addressed by means of two mechanical boundary conditions: i) Stress-free boundary condition, meaning that the normal component of the stress tensor vanishes at the surface while the displacements are not constrained, 
ii) Rigid boundary condition which demands that the components of the displacement vanish on the surface of the microcrystal. We will follow the work of Ovsyuk and Nvikov [8] to calculate the influence of a surrounding glass matrix on the breathing mode. The most remarkable facts associated with the influence of the glass matrix are: i) The presence of the glass leads to a decrease of the phonon energy when the values of the Lame's constants $\lambda$ and $\mu$ and the mass densities $\rho$ are very different between the semiconductor and glass matrix media and, ii) The appearance of a new solution associated with surface or interface modes.

In our calculations, the usual form of the MDP Hamiltonian is considered, where the main contribution is given by $D \nabla \cdot \mathbf{u}$, where $D$ is the deformation potential coupling constant. Note that, under these conditions, only longitudinal acoustic modes can couple to the carriers. For the RM, we will use the procedure illustrated by Knipp and Reinecke [6]. By considering that this electron-phonon coupling mechanism arises from a perturbation of the electronic wavefunction due to the motion of the interfaces, it can be shown that the important terms for the ripple modes are those that possess spatial dependence on the potential energy $V(\mathbf{r})$ and on the effective mass $m(\mathbf{r})$. The interaction Hamiltonian is calculated separately for each one by using an expansion in Taylor series for small acoustical displacements.

We calculate the transition rate $W$ for an electron be scattered from an initial state $i$ to a final state $f$, accompanied by the emission of an acoustical phonon characterized by the frequency $\omega_{k}$ by using the following expression

$$
W_{i \rightarrow f}=\frac{2 \pi}{\hbar}\left|M_{i \rightarrow f}(k)\right|^{2} \delta\left(E_{i}-E_{f}-\hbar \omega_{k}\right),
$$

where $M$ are the matrix elements of the electronphonon interaction involved in the transition. We use the approach proposed by Vurgaftman and Singh [10] which allow us to include the spectral broadening of the electronic spectra due to a finite energy level lifetime.

In our calculations, the electronic levels are described following the work of Ekimov et al., [11]. The material constants employed for this system are obtained through linear interpolation from the CdSe and CdS bulk parameters. We will restrict our calculations to electronic transitions between the first excited level and the ground state.

First, we calculate the scattering rates for freestanding quantum dots considering both the stress-free and the rigid boundary conditions. Our results, allow us to establish some qualitative differences in the rates due to the possible choices of the boundary conditions. A weak dependence of scattering on the mode frequency is observed in rates associated to MDP. The confined character of the breathing modes produces a reduction in the oscillations as the dot radius increases. This scattering process is practically independent of boundary condition, mainly when the spatial confinement decreases. In contrast, the relaxation rates associated to RM depend strongly on the frequency of the modes. As it should be expected, the use of the rigid boundary condition, (meaning rigid interface) neutralizes almost completely the ripple effect, which is essentially based on an macroscopic perturbation of the interfaces. Our results reproduce the behavior presented in previous works for acoustic bulk modes [6, 7], and they show that the RM is the main channel of carrier scattering in small size systems. This situation may be understood by making an analogy with the behavior of the levels in a narrow quantum well, where the position of the levels is more affected by the change in the well width (macroscopic) than for a change in the gap (microscopic).

In the Fig. 1, we shown the effect of the glass matrix on the transition rates for RM and MDP. The results also show rates for concentrations $x=0(\mathrm{CdS}), x=0.5$ and $x=1$ (CdSe).

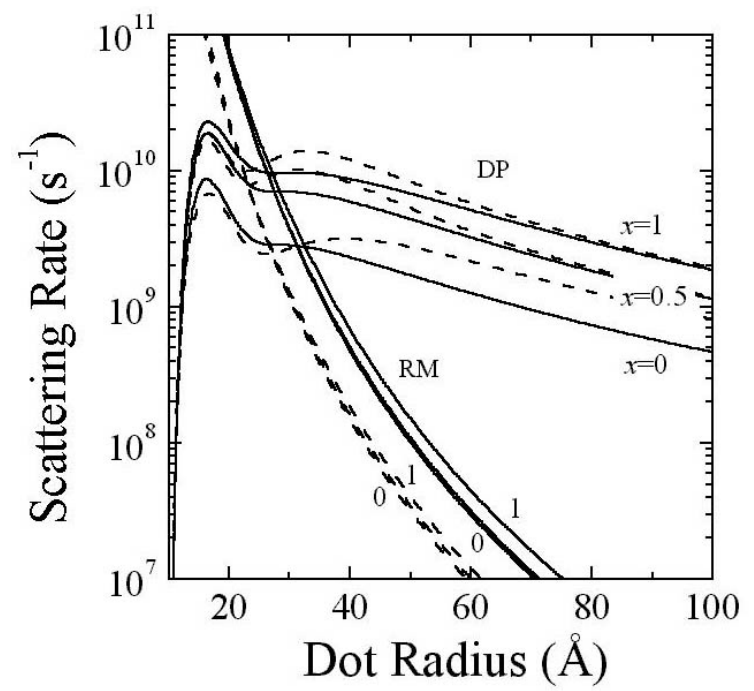

Figure 1. Electron-acoustic-phonon scattering rates in $\mathrm{CdS}_{1-x} \mathrm{Se}_{x}$ as a function of dot radius $R$. MDP and RM contributions are included. The effects of $\mathrm{SiO}_{2}\left(\mathrm{Ge}_{2}\right)$ matrix are showed in solid (dashed) lines.

Two important characteristics can be observed for the RM rate. First, as in the free-standing dots, it is observed that the ripple rates are strongly sensitive to the variations of the phonon frequencies. Now, the source of the frequency changes are related with the presence of the glass matrix. Also, for the whole range of dot radii considered, we verify that the calculated rates for dots in $\mathrm{SiO}_{2}$ matrices are larger than the rates for dots in $\mathrm{GeO}_{2}$ matrices. This last fact is a direct consequence of the softening of the phonon frequency modes when the acoustic parameters between the matrix and the quantum dot differ to each other. 
The behavior of the rates can be explained by observing that, due to the phonon normalization, both the MDP and the RM couplings depend inversely with the square root of the frequency $u \sim 1 / \sqrt{\omega}$. Therefore, a decrease in the frequency produces an increment in RM coupling and consequently an increase in their corresponding transition rates. Note that the parameter $c_{1}$ measures the degree of decrease of the breathing mode frequencies [8]. The frequency decreases when the value of $c_{1}$ becomes greater than 1 . For the $\mathrm{GeO}_{2}$, the value of the parameter $c_{1}$ is 0.99 , while for the $\mathrm{SiO}_{2}$ it is approximately 1.3 .

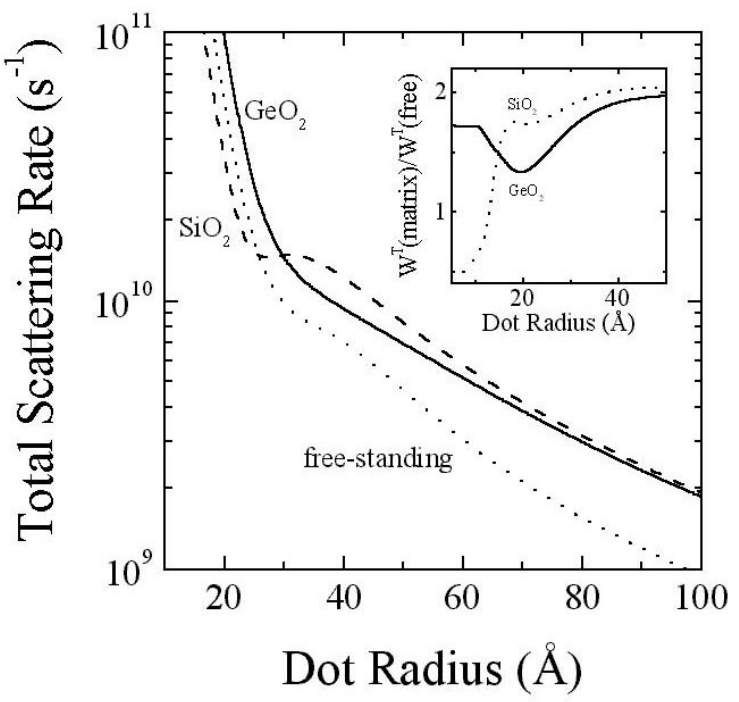

Figure 2. Total electron-acoustic-phonon scattering rates in CdSe quantum dots. Stress-free boundary condition was used in this calculation. Inset shows the ratio between the total rate for a CdSe dot embedded in a glass matrix $\left[W^{T}\right.$ (matrix)] and the total rate for a free-standing dot $\left[W^{T}\right.$ (free) $]$.

In Fig. 2 we show the dependence of the total scattering rates as a function of the radius of the CdSe quantum dot for two different glass matrices $\left(\mathrm{SiO}_{2}\right.$, $\mathrm{GeO}_{2}$ ). The total scattering rate for a free-standing dot is also represented in dotted lines. Stress-free boundary conditions were used for this calculation. The rapid increase of the total scattering rates in the small dot sizes regime is due to the influence of the RM. This effect could explain the results of Klimov et al. [13], who has observed that at small dots (radii less than $40 \AA$ ) the measured relaxation times increase linearly with the radius. This observation cannot be explained from arguments that include relaxation assisted by optical phonons. The effect of the glass matrix can be better illustrated in the inset of the Fig. 2, where we display the ratio of the total scattering rate for a CdSe dot embedded in a glass matrix to the total scattering rate for a free-standing dot $\left[W^{T}\right.$ (matrix) $/ W^{T}$ (free) $]$. In general, absolute variations of the order $75-100 \%$ can be observed. We can also observe that for small dot sizes, where the contribution of the RM on the total rate is dominant, the effects induced by the glass matrix are more visible. However, for size dots greater than $80 \AA$, the effect of the matrix and therefore of the RM becomes almost negligible.

Although we considered only breathing modes in this work, the above considerations can be extended to other phonon modes. However, we believe that if we consider acoustic modes with $l>0$, the relative importance of the RM should become gradually smaller, since the acoustic frequencies increase linearly with the angular moment $l$. This change in the mode frequencies would produce a smaller coupling via RM.

In conclusion, we note that the main scattering channel for acoustic modes arises mainly through the MDP. Our results also demonstrate that the inclusion of the RM should be considered mainly in small dot size systems. Finally, it is clear that the surrounding material plays a significant role in the electron-acousticphonon coupling, and its effects should be included in the analysis of the relaxation processes.

\section{Acknowledgments}

This work has been supported by Fundação de Amparo à Pesquisa do Estado de São Paulo (FAPESP), Conselho de Desenvolvimento Científico e Tecnológico (CNPq), PEPCI/USF and by the U.S. Office of Naval Research.

\section{References}

[1] A. M. Alcalde, G. E. Marques, G. Weber, and T. L. Reinecke, Solid State Commun. 116, 247 (2000).

[2] A. M. Alcalde and G. Weber, Semicond. Sci. Technol. 15, 1082 (2000).

[3] T. Takagahara, J. Lumin. 70, 129 (1996).

[4] E. Duval, A. Boukenter, and A. Champagnon, Phys. Rev. Lett. 56, 2052 (1986).

[5] L. Saviot et al., J. Non-Crystalline Solids 197, 238 (1996).

[6] P. A. Knipp and T. L. Reinecke, Phys. Rev. B 52, 5923 (1995).

[7] F. T. Vasko and V. Mitin, Phys. Rev. B 52, 1500 (1995).

[8] N. N. Ovsyuk and V. N. Novikov, Phys. Rev. B 53, 3113 (1996).

[9] A. Tamura, K. Higeta, and T. Ichinokawa, J. Phys. C: Solid State Phys. 15, 4975 (1982).

[10] I. Vurgaftman and J. Singh, Appl. Phys. Lett. 64, 232 (1994).

[11] A. I. Ekimov et al., J. Opt. Soc. Am. B 10, 100 (1993).

[12] U. Bockelmann and G. Bastard, Phys. Rev. B 42, 8947 (1990).

[13] V. I. Klimov, D. W. McBranch, C. A. Leatherdale, and M. G. Bawendi, Phys. Rev. B 60, 13740 (1999). 